\title{
Tuberculous pleural effusion
}

\author{
Kan Zhai, Yong Lu, Huan-Zhong Shi \\ Department of Respiratory and Critical Care Medicine, Beijing Institute of Respiratory Medicine and Beijing Chaoyang Hospital, Capital Medical \\ University, Beijing 100020, China \\ Contributions: (I) Conception and design: HZ Shi; (II) Administrative support: None; (III) Provision of study materials or patients: None; (IV) \\ Collection and assembly of data: None; (V) Data analysis and interpretation: None; (VI) Manuscript writing: All authors; (VII) Final approval of \\ manuscript: All authors. \\ Correspondence to: Huan-Zhong Shi, MD, PhD. Department of Respiratory and Critical Care Medicine, Beijing Chaoyang Hospital, Capital Medical \\ University, 8 Gongti Nanlu, Chaoyang District, Beijing 100020, China. Email: shihuanzhong@sina.com.
}

\begin{abstract}
Although it is curable, tuberculosis remains one of the most frequent causes of pleural effusions on a global scale, especially in developing countries. Tuberculous pleural effusion (TPE) is one of the most common forms of extrapulmonary tuberculosis. TPE usually presents as an acute illness with fever, cough and pleuritic chest pain. The pleural fluid is an exudate that usually has predominantly lymphocytes. The gold standard for the diagnosis of TPE remains the detection of Mycobacterium tuberculosis in pleural fluid, or pleural biopsy specimens, either by microscopy and/or culture, or the histological demonstration of caseating granulomas in the pleura along with acid fast bacilli, Although adenosine deaminase and interferon- $\gamma$ in pleural fluid have been documented to be useful tests for the diagnosis of TPE. It can be accepted that in areas with high tuberculosis prevalence, the easiest way to establish the diagnosis of TPE in a patient with a lymphocytic pleural effusion is to generally demonstrate a adenosine deaminase level above $40 \mathrm{U} / \mathrm{L}$. The recommended treatment for TPE is a regimen with isoniazid, rifampin, and pyrazinamide for two months followed by four months of two drugs, isoniazid and rifampin.
\end{abstract}

Keywords: Pleural effusion; tuberculous pleurisy; tuberculosis

Submitted Apr 22, 2016. Accepted for publication Apr 30, 2016.

doi: $10.21037 /$ jtd.2016.05.87

View this article at: http://dx.doi.org/10.21037/jtd.2016.05.87

\section{Introduction}

Tuberculosis accounts for millions of active disease cases and deaths in both developed and developing countries. According to the World Health Organization, there were an estimated 9.6 million incident cases of tuberculosis globally in 2014: 5.4 million among men, 3.2 million among women and 1.0 million among children (1). Tuberculous pleural effusion (TPE) results from Mycobacterium tuberculosis infection of the pleura and is characterized by an intense chronic accumulation of fluid and inflammatory cells in pleural space (2). So far, no formal guidelines are available for diagnosis and treatment of tuberculous pleurisy. In order to provide the evidence-based updated information concerning diagnosis and treatment of TPE for general physician, specialists, and primary care providers, we therefore write the current review article.

\section{Incidence}

TPE is the second most common form of extrapulmonary tuberculosis and a common cause of pleural effusions in endemic tuberculosis areas (2). The proportion of patients with tuberculosis who have pleural effusions has varied markedly from population to population. In the United States, although the total number of patients with TPE decreased between 1993 and 2003, the proportion of patients with TPE compared to the total number of tuberculosis cases remains relatively stable (median proportion, $3.6 \%$ ) (3). In contrast more than $25 \%$ of patients with tuberculosis in Burundi are reported to have TPE (4). In South Africa, 20\% of tuberculosis patients have TPE (5), while in Spain, this proportion is greater than 16\% (6). The percentage of patients with pulmonary tuberculosis also had TPE is higher in human immunodeficiency virus 
Table 1 The proportion of TPE in total pleural effusions diagnosed by medical thoracoscopy

\begin{tabular}{lcc}
\hline Country & $\mathrm{n}$ & TPE (\%) \\
\hline China (10) & 833 & 40.0 \\
South Africa (11) & 51 & 82.4 \\
India (12) & 68 & 23.5 \\
New Zealand (13) & 51 & 5.9 \\
United Kingdom (14) & 56 & 5.6 \\
Spain (15) & 93 & 3.2 \\
France (16) & 149 & 2.7 \\
Denmark (17) & 146 & 2.1 \\
United States (18) & 51 & 0 \\
\hline
\end{tabular}

TPE, tuberculous pleural effusion.

(HIV)-positive than in HIV-negative patients in South Africa (38\% vs. 20\%) (5), Zimbabwe (27\% vs. $13 \%$ ) (7), and Uganda (23\% vs. $11 \%)(8)$.

Tuberculosis is always the leading etiology of pleural effusions in the developing countries (9). For example, in the largest series of 833 Chinese patients with undiagnosed pleural effusion who undergo medical thoracoscopy, 40.0\% of cases are confirmed to suffer from TPE (10). As shown in Table 1, relative high percentages of TPE in pleural effusions diagnosed by medical thoracoscopy were also seen the other high tuberculosis burden countries, such as South Africa (82.4\%) (11), India (23.5\%) (12). In contrast, the proportions of TPE are very low in the low tuberculosis settings, such as New Zealand (5.9\%) (13), United Kingdom (5.6\%) (14), Spain (3.2\%) (15), France (2.7\%) (16), Denmark (2.1\%) (17), and United States (0\%) (Table 1) (18).

\section{Clinical manifestations}

TPE predominates in men, with an overall male-to-female ratio of 2:1 (19). In an epidemiological analysis from the United States, TPE occurs significantly more often than pulmonary tuberculosis among persons $>65$ years old, and the mean age of patients with TPE is 49 years: about $50 \%$ were younger than 45 years and $30 \%$ were over 65 years of age (3). In contrast, TPE affects mainly younger individuals (mean age $=34$ years) in higher tuberculosis burden areas, where primary infection accounts for a large percentage of patients with TPE (19).

TPE usually manifests as an acute illness, especially in younger patients who are more immunocompetent. Upon presentation symptoms in one series had been present for less than a month $50 / 71$ patients $(71 \%)$ and had been present for less than 1 week in $25 / 71$ patients (35\%) (20). The most frequent symptoms of TPE are nonproductive cough and pleuritic chest pain; if both cough and chest pain are present, the pain usually precedes the cough (21-23). Most patients are febrile but approximately $15 \%$ will be afebrile. Dyspnea can be present in some TPE patients if the effusion is large. Other symptoms include night sweats, weight loss, malaise (2).

TPE is usually unilateral and can be of any size. In our series of 333 patients, pleural fluid occurred only on the left side in 127 (38.1\%), only on the right in 161 (48.4\%), and both sides were affected in 45 (13.5\%) (23). In either unilateral or bilateral effusion, the percentages of small, moderate, and large size of pleural effusions were 20.4\%, $19.2 \%$, and $60.4 \%$, respectively (23). Approximately $20 \%$ of patients with TPE have coexisting parenchymal disease on chest radiograph (24). However, computed tomography scanning offers a more sensitive method and can demonstrate parenchymal disease in $40-85 \%$ of cases $(23,25,26)$.

\section{Diagnosis}

The definitive diagnosis of TPE depends on the demonstration of Mycobacterium tuberculosis in the sputum, pleural fluid, or pleural biopsy specimens $(2,27)$. The presumptive diagnosis can also be established with reasonable certainty by demonstrating granuloma in the parietal pleura or elevated concentrations of adenosine deaminase (ADA) or interferon- $\gamma$ in pleural fluid in the adequate clinical context $(2,28)$.

\section{Tuberculin skin tests}

In areas of low tuberculosis prevalence, or no vaccination, a positive tuberculin skin test result is supportive evidence in the diagnosis of TPE; however, a negative result can be seen in approximately one third of patients (24). Since a negative test does not rule out the diagnosis of TPE, tuberculin skin test is being utilized less and less in patients suspected of having TPE, especially in high tuberculosis burden countries. In addition, if the patient is markedly immunosuppressed with HIV infection or is severely malnourished, the skin test is always negative (27).

\section{Mycobacterial stain and culture}

It is necessary to obtain sputum in addition to pleural fluid 
for the acid-fast bacilli smear and Mycobacteria tuberculosis culture in patients with suspected TPE, even in the absence of parenchymal involvement. Conde $e t$ al. prospectively evaluated the diagnostic yield of mycobacterial smears and cultures in 84 patients with TPE, and found that the induced sputum was smear positive in $12 \%$ and culturepositive in $52 \%$ of cases (29). Direct examination of pleural fluid for acid-fast bacilli in immunocompetent individuals are not indicated because they are almost always negative unless the patient has a tuberculous empyema (24). Acidfast bacilli can be found in approximately $20 \%$ of HIV positive individuals, smears therefore should be obtained in immunocompromised hosts (27).

In a series of 254 patients with TPE, 93 patients (36.6\%) had positive of pleural fluid cultures for Mycobacteria tuberculosis in the Löwenstein-Jensen medium (24). Thus, pleural fluid cultures should be performed in any patient with an undiagnosed pleural effusion. The combination of pleural fluid and sputum cultures in the diagnostic workup of TPE seems a reasonable initial approach, with a combined diagnostic yield of $79 \%(30,31)$.

\section{Adenosine deaminase}

ADA is a predominant T-lymphocyte enzyme that catalyzes the conversion of adenosine and deoxyadenosine to inosine and deoxyinosine, respectively. Many studies have investigated the usefulness of $\mathrm{ADA}$ in pleural fluid for the early diagnosis of TPE, and confirmed that the determination of ADA levels is an easy and inexpensive method for diagnosing TPE. ADA levels in pleural fluid are also elevated in HIV patients even with very low $\mathrm{CD}^{+} \mathrm{T}$ cells (32). Our meta-analysis involving 63 studies demonstrated that the summary receiver operating characteristic curves for the sensitivity and specificity of ADA in pleural fluid in the diagnosis of TPE were $92 \%$ and $90 \%$, respectively. The positive likelihood ratio was 9.03 , the negative likelihood ratio was 0.10 , and the diagnostic odds ratio was 110.08 (33).

Almost all patients with TPE have a pleural fluid ADA level above $40 \mathrm{U} / \mathrm{L}$, which is the most widely accepted cutoff value for the diagnosis of TPE (2). The higher the level, the greater the chance of the patient having TPE while the lower the level the lesser the chance of the patient having TPE (34). High level of ADA can also be seen in pleural effusions with the other etiologies. About two-thirds of empyemas and one-third of parapneumonic effusions have ADA levels above $40 \mathrm{U} / \mathrm{L}$, however, the differential diagnosis of TPE from these conditions is not difficult, since both clinical picture and polymorphonuclear leukocytes predominant pleural fluid of patients with parapneumonic effusions and empyemas are total different from those of patients with TPE. There are several other instances in which high pleural fluid ADA has been reported in brucellosis, Q fever, lymphomas, rheumatoid arthritis, etc. $(2,25)$.

ADA has two molecular forms, ADA1 and ADA2. ADA1 is found in all cells and has it greatest activity in lymphocytes and monocytes while ADA2 is found only in monocytes (7). The ADA2 isoenzyme is primarily responsible for the increased total ADA activity in TPE, whereas high ADA in nontuberculous effusions is due to an increase in the ADA1 isoenzyme. Nevertheless, although the determination of the ADA2 isoenzyme is at least as sensitive and more specific than that of total ADA activity for diagnosing TPE (35). However, because the additional yield is small, a study would require a very large sample size to demonstrate that isoenzymes have significantly higher specificity than total ADA activity.

Currently, pleural fluid $\mathrm{ADA}$ is routinely employed in the diagnostic workup of pleural effusions in high tuberculosis burden countries. However, the negative predictive value remains high even though the positive predictive value of pleural ADA declines in the developed countries. Therefore, when interpreting ADA levels, the clinician must additionally be aware of situations which may increase the likelihood of both the false-negative and false-positive ADA results.

\section{Interferon-gamma}

Interferon- $\gamma$ is a cytokine released by activated $\mathrm{CD} 4^{+} \mathrm{T}$ cells that increases the mycobactericidal activity of macrophages. Elevated level of interferon- $\gamma$ in TPE was reported for the first time in 1988 (36). In a meta-analysis of 22 studies that included 782 patients with TPE and 1,319 patients with nontuberculous effusions, we have provided the evidence to show that the overall sensitivity of the interferon- $\gamma$ assay was $89 \%$, the overall specificity was $97 \%$, and the maximum joint sensitivity and specificity was $95 \%$ (37). Based on the comparison of the related diagnostic measures, including sensitivity, specificity, positive likelihood ratio, negative likelihood ratio, and diagnostic odds ratio, the overall accuracy of interferon- $\gamma$ determination for the diagnosis of TPE seems to be somehow better than that of ADA (37). However, the long historical success of ADA and the fact that it is simpler and less expensive that the interferon- $\gamma$ test 
makes it the preferred test.

In vitro $\mathrm{T}$-cell-based interferon- $\gamma$ release assays have been developed and licensed for diagnosis of latent tuberculosis for a couple of years. Normally, these tests are T cell based in vitro assays that measure interferon- $\gamma$ release by sensitized $\mathrm{T}$ cells from peripheral blood or pleural fluid in response to highly Mycobacterium tuberculosis-specific antigens such as early secretory antigen-6 and culture filtrate protein-10 (28). There are now two commercially available interferon- $\gamma$ release assays (QuantiFERON-TB Gold and T-SPOT.TB) for clinical use. These tests are good at identifying patients who have been infected with Mycobacterium tuberculosis, but they are much less useful in identifying patients with TPE. Our recent two meta-analyses indicated that the overall performances of fluid and blood interferon- $\gamma$ release assays for the diagnosis of TPE are not as high as expected and varied by fluid category $(38,39)$. With regard to its high cost, sophisticated technique, and difficulty in interpretation, are not recommended on either the blood or the pleural fluid to make a diagnosis of TPE.

\section{Nucleic acid amplification tests}

Several commercial and in-house nucleic acid amplification tests are available for the amplification and detection of Mycobacterium tuberculosis-specific nucleic acid sequences from specimens such as sputum and pleural fluid. A pooled analysis of the data from 20 studies assessing the use of pleural fluid nucleic acid amplification tests concluded that that these tests demonstrated reasonably high specificity ( $97 \%$ for commercial and $91 \%$ for in-house tests) but generally poor and variable sensitivity (62\% for commercial and $76.5 \%$ for in-house tests) for diagnosing TPE (40). Very similar results were obtained in another systematic review and meta-analysis of 40 studies (41). It is believed that the disappointingly low sensitivities of nucleic acid amplification techniques might be due to: (I) the presence of inhibitory substances in pleural fluid; (II) the small amount of Mycobacterium tuberculosis in pleural fluid; (III) technical defect of nucleic acid extraction. Therefore, thus, the inconsistent results of different studies and the high cost of nucleic acid amplification tests limit their use to investigational settings.

\section{Other biochemical tests}

Many studies have evaluated the diagnostic accuracy of additional pleural fluid assays and biomarkers for the management of TPE, these biomarkers include cell subsets, B-cell response, complement activation, neopterin, leptin, lysozyme, and the other cytokines, etc. (28). The current evidence does not support the use of none of the above biomarkers for the diagnosis of TPE because of their low sensitivity or/and specificity.

Surprisingly, we have reported for the first time that the concentrations of interleukin 27 in TPE are significantly higher than those in pleural effusions with the other etiologies, and that pleural interleukin 27 levels can be used for diagnosing TPE, with sensitivity of $92.7 \%$ and specificity of $98.8 \%$ (42). The quite good diagnostic performance of interleukin 27 differentiating TPE from the other pleural effusions have been confirmed another two studies from China $(43,44)$. Two studies from low tuberculosis prevalence settings also show that the product of interleukin 27 and ADA has better sensitivity than ADA alone or ADA2, respectively, for diagnosing TPE $(45,46)$. Further studies are needed to elucidate the role of interleukin 27 in this process.

\section{Pleural biopsy}

One key criticism of using soluble biomarkers rather than culture or biopsy examinations for the diagnosis of TPE is that none of the biomarkers provide definite etiologic diagnosis. It can only be accepted that a definite diagnosis of TPE is achieved when Mycobacterium tuberculosis is demonstrated in sputum or pleural specimens, or when epithelioid cell granulomas and/or caseating granulomas are found in pleural biopsies. The most common procedure to establish the definite diagnosis of TPE over the past several decades has been with a blind needle biopsy of the pleura. In one study of 248 patients with TPE, closed needle biopsy of the pleura revealed granulomas in $80 \%$ of patients, acid-fast bacilli stain was positive in $25.8 \%$ and Mycobacteria tuberculosis culture was positive in $56 \%$ of patients (24).

It should be mentioned that computed tomography- or ultrasound-guided pleural biopsies have been demonstrated to be helpful for the investigation of pleural effusions $(47,48)$; and it has been suggested the image-guided pleural needle biopsy can be used as the primary method of diagnosis in patients with pleural thickening identified by computed tomography which can be targeted.

Differential diagnosis of TPE sometimes mandates more invasive procedures like medical thoracoscopy when one or more thoracenteses or/and blind needle biopsy fail to reach definite diagnosis. A recent meta-analysis revealed 
Table 2 Recommended doses of first-line anti-tuberculosis drugs for adults*

\begin{tabular}{|c|c|c|c|c|}
\hline \multirow{3}{*}{ Drug } & \multicolumn{4}{|c|}{ Recommended dose } \\
\hline & \multicolumn{2}{|l|}{ Daily } & \multicolumn{2}{|l|}{3 times per week } \\
\hline & Dose and range (mg/kg body weight) & Maximum (mg) & Dose and range (mg/kg body weight) & Daily maximum (mg) \\
\hline Rifampin & $10[8-12]$ & 600 & $10[8-12]$ & 600 \\
\hline Pyrazinamide & 25 [20-30] & - & $35[30-40]$ & - \\
\hline
\end{tabular}

*, adapted from World Health Organization. Guidelines for treatment of tuberculosis, Fourth edition, 2010 (52).

that there is no mortality with medical thoracoscopy, and that the rates of major and minor complications are $1.5 \%$ and $10.5 \%$, respectively, indicating that is a very safe procedure in the diagnosis of exudative pleural effusions. In the largest series of 333 patients with TPE, our data showed that medical thoracoscopy with pleural biopsies yielded tuberculosis pathology in 330 (99.1\%) patients by demonstration of one or more of following histological abnormalities in pleural biopsy specimen: (I) acid-fast bacillus; (II) caseating granulomas; (III) epithelioid cell granuloma with no evidence of other granuloma diseases.

\section{Treatment}

In many cases, TPE is a self-limited disease, and the natural history of an untreated TPE is characterized by spontaneous resolution in 4 to 16 weeks; however, about half of untreated patients would subsequently develop some form of the active disease $(49,50)$. The goals of the treatment of TPE includes: (I) to prevent the subsequent occurrence of active tuberculosis; (II) to relieve the symptoms of TPE; and (III) to avoid the presence of a fibrothorax.

With appropriate treatment, the symptoms and radiological abnormalities of patients with TPE gradually abate. The typical patient becomes afebrile within two weeks, but temperature elevations may persist as long as two months (51). The mean time for the complete resorption of pleural fluid is approximately 6 weeks, but it can be as long as 12 weeks. There is no reason to keep the patient at bed rest and the patient needs to be isolated only if their sputum is positive for Mycobacteria tuberculosis.

\section{Anti-tuberculosis chemotherapy}

Anti-tuberculosis chemotherapy of TPE should be the same as that used for pulmonary tuberculosis (52). The patients with TPE should be treated with isoniazid, rifampin, and pyrazinamide for 2 months followed by 4 months of two drugs, isoniazid and rifampin (2HRZ/4HR) (Table 2) (52,53). Directly observed therapy is recommended, since $16 \%$ to $49 \%$ of overall patients do not complete the regimen (54).

The emergence of drug-resistant tuberculosis, especially multidrug-resistant and extensively drugresistant tuberculosis, has posed a significant threat to the treatment of all forms of tuberculosis (55). In China, $34.2 \%$ of the new cases of tuberculosis and $54.5 \%$ of the previously treated cases were resistant to at least one of the first-line anti-tuberculosis drugs; $5.7 \%$ of new cases and $25.6 \%$ of previously treated cases were multidrugresistant tuberculosis (56). In the United States, $9.9 \%$ of patients have isolates resistant to at least one first-line antituberculosis drug, whereas the rate of multidrug resistance is detected in $1 \%$ of cases (3).

Therefore, it is reasonable to include ethambutol in the treatment regimen for TPE, although it is likely that the standardized therapy without this drug would be a valid treatment option, unless there is little possibility of drug resistance. Once susceptibility to isoniazid, rifampin, and pyrazinamide has been confirmed, ethambutol can be discontinued. In young children with TPE, ethambutol is always omitted if the source of transmission is known to have drug-susceptible tuberculosis, because the toxic effects of ethambutol is more concern in children. Nine-month regimens using isoniazid and rifampin are also effective when the organisms are fully susceptible to the drug.

In the case of resistance to isoniazid, but not to rifampin, a similar anti-tuberculous treatment outcome can be expected by using a standard 6-month regimen in which isoniazid is replaced by a later generation fluoroquinolone (levofloxacin or moxifloxacin) (53). In addition, a 6-month regimen containing rifampin, moxifloxacin, pyrazinamide, 
and ethambutol for 2 months, followed by rifapentine and moxifloxacin for 4 months, has also been shown to be effective (57).

When TPE is caused by multidrug-resistant Mycobacteria tuberculosis confirmed by either by culture or with the use of DNA-based methods, the initial treatment regimen should be individually tailored according to the results of drugsusceptibility testing. In the absence of this information, empirical regimens can be used, but as soon as the results of drug susceptibility testing become available, the treatment regimen should be adjusted (58). According to the results of an individual patient data meta-analysis, the World Health Organization recommends that the initial treatment regimen for multidrug-resistant tuberculosis include four drugs to which the patient's isolate is susceptible (plus pyrazinamide, for which susceptibility results are not usually available) in the induction phase, which should last 6 to 8 months (59). The World Health Organization also recommends a minimum of 20 months (including the induction phase), but this recommendation is based on a database that included few patients treated for shorter periods.

\section{Therapeutical thoracentesis}

If the patient has dyspnea because of a large pleural effusion, a therapeutical thoracentesis is recommended. It should be noted that complete clearance of pleural fluid pigtail drainage to an effective anti-tuberculosis regimen does not appear significantly to decrease the incidence of residual pleural thickening and other clinical symptom (60).

\section{Fibrinolytics}

At the end of anti-tuberculosis treatment, approximately $25 \%$ of patients with TPE show residual pleural thickening $(1 \mathrm{~cm})$; however, this decreases with time and has negligible functional consequences (61). The administration of a fibrinolytic may decrease the degree of residual pleural thickening in patients with loculated TPEs. Instillation of fibrinolytics, in addition to anti-tuberculosis medication, in patients with symptomatic loculated tuberculosis effusions may hasten the resolution of pleural effusion and reduce the incidence of residual pleural thickening (62).

\section{Corticosteroids}

The role of corticosteroids in the treatment of TPE is controversial. In two controlled studies in which therapeutical thoracentesis was performed there were no benefits $(63,64)$. In a third study in which no therapeutical thoracentesis was performed, the duration of fever and the time required for fluid resorption were decreased (65). A recent Cochrane review concluded that there are insufficient data to support evidence-based recommendations regarding the use of adjunctive corticosteroids in people with TPE (66). Nevertheless, in selected patients who continue to have severe systemic symptoms (e.g., fever, malaise, pleuritic chest pain) after two weeks of anti-tuberculosis treatment and therapeutic thoracentesis, a short course of corticosteroids may be beneficial.

There are concerns regarding the use of corticosteroids in HIV-positive individuals due to the possibility of increased risk of opportunistic infections. In one randomized study of 197 patients with HIV associated TPE, the administration of prednisolone was associated with an increased risk of Kaposi sarcoma (67). Because there is a lack of survival benefit and increased risk of Kaposi sarcoma, the use of steroids in HIV associated TPE is not currently recommended.

\section{Treatment of tuberculous pleural effusion (TPE) in patients with human immunodeficiency virus (HIV) infection}

The treatment of TPE in patients with HIV infection should be treated similarly to those who are HIVnegative. TPE patients with HIV infection should receive antiretroviral therapy while they are receiving treatment for tuberculosis. If patients are not already receiving antiretroviral therapy when tuberculosis is diagnosed, antiretroviral therapy should be initiated within two weeks after starting anti-tuberculosis treatment for persons with a CD4 $4^{+}$T-cell count of less than or equal to 50 per cubic millimeter and within 8 weeks for persons with a count above 50 per cubic millimeter $(68,69)$. However, drug interactions between highly active antiretroviral therapy and anti-tuberculosis therapy are a significant concern. In addition, the clinician should also pay attention to adverse drug reactions, paradoxical reactions, and the immune reconstitution inflammatory syndrome; the latter is manifested by an increase in the volume of existing pleural fluid or worsening symptoms (70).

\section{Tuberculous empyema}

Tuberculous empyema represents an uncommon chronic, 
active infection of the pleural space, which is characterized by the presence of thick pus and the visceral pleura is usually calcified (71). Acid-fast bacilli smears and cultures are usually positive. Penetration of anti-tuberculosis drugs is impaired, and surgical drainage is often needed to control the situation. In addition to a standard anti-tuberculosis regimen, these patients may require serial thoracocentesis, extrapleural pneumonectomy or thoracoscopy.

\section{Recommendations}

If pleural effusion is lymphocytic predominant, pleural fluid ADA can be used as a screening test regardless of the prevalence of tuberculosis in the specific geographical area (72). If pleural ADA is above $40 \mathrm{U} / \mathrm{L}$ with lymphocyte-to-neutrophil ratio of more than 0.75 , a presumptive diagnosis of TPE can be made (9). Nevertheless, if the patient's clinical picture is not typical for TPE; consideration can be given to performing a needle biopsy of the pleura, or medical thoracoscopy, or open pleural biopsy.

An ADA level less than $40 \mathrm{U} / \mathrm{L}$ virtually rules out TPE and no further invasive diagnostic procedures should be necessary for diagnosing TPE. However, if the patient has a typical clinical presentation of tuberculosis (i.e., febrile young patient from an endemic area of tuberculosis with a negative pleural fluid cytological investigation), especially with lymphocytic predominant pleural fluid, the possibility of TPE can be further evaluated with needle biopsy of the pleura, or medical thoracoscopy, or open pleural biopsy (2).

Once the definite diagnosis of TPE is established, the above mentioned standard treatment regimen with isoniazid, rifampin, and pyrazinamide for 2 months followed by 4 months of two drugs, isoniazid and rifampin (2HRZ/4HR) can be stated. In some situations, even when a presumptive diagnosis of TPE is made, anti-tuberculosis chemotherapy should also be initiated, provided that the diagnosis occurs in a country with a moderate or high incidence of tuberculosis and low drug-resistance rates.

\section{Acknowledgements}

None.

\section{Footnote}

Conflicts of Interest: The authors have no conflicts of interest to declare.

\section{References}

1. Global tuberculosis report 2015. Geneva: World Health Organization, 2015. Available online: http://apps.who. int/iris/bitstream/10665/191102/1/9789241565059_eng. pdf?ua=1, accessed 18 April 2016

2. Light RW. Pleural diseases. 6th ed. Philadelphia: Lippincott Williams \& Wilkins, 2013.

3. Baumann MH, Nolan R, Petrini M, et al. Pleural tuberculosis in the United States: incidence and drug resistance. Chest 2007;131:1125-32.

4. Mlika-Cabanne N, Brauner M, Kamanfu G, et al. Radiographic abnormalities in tuberculosis and risk of coexisting human immunodeficiency virus infection. Methods and preliminary results from Bujumbura, Burundi. Am J Respir Crit Care Med 1995;152:794-9.

5. Saks AM, Posner R. Tuberculosis in HIV positive patients in South Africa: a comparative radiological study with HIV negative patients. Clin Radiol 1992;46:387-90.

6. Valdés L, Ferreiro L, Cruz-Ferro E, et al. Recent epidemiological trends in tuberculous pleural effusion in Galicia, Spain. Eur J Intern Med 2012;23:727-32.

7. Pozniak AL, MacLeod GA, Ndlovu D, et al. Clinical and chest radiographic features of tuberculosis associated with human immunodeficiency virus in Zimbabwe. Am J Respir Crit Care Med 1995;152:1558-61.

8. Awil PO, Bowlin SJ, Daniel TM. Radiology of pulmonary turberculosis and human immunodeficiency virus infection in Gulu, Uganda. Eur Respir J 1997;10:615-8.

9. Light RW. Update on tuberculous pleural effusion. Respirology 2010;15:451-8.

10. Wang XJ, Yang $Y$, Wang Z, et al. Efficacy and safety of diagnostic thoracoscopy in undiagnosed pleural effusions. Respiration 2015;90:251-5.

11. Diacon AH, Van de Wal BW, Wyser C, et al. Diagnostic tools in tuberculous pleurisy: a direct comparative study. Eur Respir J 2003;22:589-91.

12. Prabhu VG, Narasimhan R. The role of pleuroscopy in undiagnosed exudative pleural effusion. Lung India 2012;29:128-30.

13. Wilsher ML, Veale AG. Medical thoracoscopy in the diagnosis of unexplained pleural effusion. Respirology 1998;3:77-80.

14. Munavvar M, Khan MA, Edwards J, et al. The autoclavable semirigid thoracoscope: the way forward in pleural disease? Eur Respir J 2007;29:571-4.

15. Ferrer J, Roldan J, Teixidor J, et al. Predictors of pleural malignancy in patients with pleural effusion undergoing 
thoracoscopy. Chest 2005;127:1017-22.

16. Blanc FX, Atassi K, Bignon J, et al. Diagnostic value of medical thoracoscopy in pleural disease: a 6-year retrospective study. Chest 2002; 121: 1677-83.

17. Hansen M, Faurschou P, Clementsen P. Medical thoracoscopy, results and complications in 146 patients: a retrospective study. Respir Med 1998;92:228-32.

18. DePew ZS, Wigle D, Mullon JJ, et al. Feasibility and safety of outpatient medical thoracoscopy at a large tertiary medical center: a collaborative medical-surgical initiative. Chest 2014;146:398-405.

19. Porcel JM. Tuberculous pleural effusion. Lung 2009;187:263-70.

20. Levine H, Szanto PB, Cugell DW. Tuberculous pleurisy. An acute illness. Arch Intern Med 1968;122:329-32.

21. Berger HW, Mejia E. Tuberculous pleurisy. Chest 1973;63:88-92.

22. Seibert AF, Haynes J Jr, Middleton R, et al. Tuberculous pleural effusion. Twenty-year experience. Chest 1991;99:883-6.

23. Wang Z, Xu LL, Wu YB, et al. Diagnostic value and safety of medical thoracoscopy in tuberculous pleural effusion. Respir Med 2015;109:1188-92.

24. Valdés L, Alvarez D, San José E, et al. Tuberculous pleurisy: a study of 254 patients. Arch Intern Med 1998;158:2017-21.

25. Yilmaz MU, Kumcuoglu Z, Utkaner G, et al. Computed tomography findings of tuberculous pleurisy. Int J Tuberc Lung Dis 1998;2:164-7.

26. Kim HJ, Lee HJ, Kwon SY, et al. The prevalence of pulmonary parenchymal tuberculosis in patients with tuberculous pleuritis. Chest 2006;129:1253-8.

27. Gopi A, Madhavan SM, Sharma SK, et al. Diagnosis and treatment of tuberculous pleural effusion in 2006. Chest 2007;131:880-9.

28. Trajman A, Pai M, Dheda K, et al. Novel tests for diagnosing tuberculous pleural effusion: what works and what does not? Eur Respir J 2008;31:1098-106.

29. Conde MB, Loivos AC, Rezende VM, et al. Yield of sputum induction in the diagnosis of pleural tuberculosis. Am J Respir Crit Care Med 2003;167:723-5.

30. Ruan SY, Chuang YC, Wang JY, et al. Revisiting tuberculous pleurisy: pleural fluid characteristics and diagnostic yield of mycobacterial culture in an endemic area. Thorax 2012;67:822-7.

31. von Groote-Bidlingmaier F, Koegelenberg CF, Bolliger CT, et al. The yield of different pleural fluid volumes for Mycobacterium tuberculosis culture. Thorax 2013;68:290-1.
32. Baba K, Hoosen AA, Langeland N, et al. Adenosine deaminase activity is a sensitive marker for the diagnosis of tuberculous pleuritis in patients with very low CD4 counts. PloS One 2008;3:e2788.

33. Liang QL, Shi HZ, Wang K, et al. Diagnostic accuracy of adenosine deaminase in tuberculous pleurisy: a metaanalysis. Respir Med 2008;102:744-54.

34. Valdés L, Alvarez D, Valle JM, et al. The etiology of pleural effusions in an area with high incidence of tuberculosis. Chest 1996;109:158-62.

35. Zemlin AE, Burgess LJ, Carstens ME. The diagnostic utility of adenosine deaminase isoenzymes in tuberculous pleural effusions. Int J Tuberc Lung Dis 2009;13:214-20.

36. Ribera E, Ocana I, Martinez-Vazquez JM, et al. High level of interferon gamma in tuberculous pleural effusion. Chest 1988;93:308-11.

37. Jiang J, Shi HZ, Liang QL, et al. Diagnostic value of interferon-gamma in tuberculous pleurisy: a metaanalysis. Chest 2007;131:1133-41.

38. Zhou Q, Chen YQ, Qin SM, et al. Diagnostic accuracy of T-cell interferon-gamma release assays in tuberculous pleurisy: a meta-analysis. Respirology 2011;16:473-80.

39. Zhou XX, Liu YL, Zhai K, et al. Body Fluid Interferongamma Release Assay for Diagnosis of Extrapulmonary Tuberculosis in Adults: A Systematic Review and MetaAnalysis. Sci Rep 2015;5:15284.

40. Dinnes J, Deeks J, Kunst H, et al. A systematic review of rapid diagnostic tests for the detection of tuberculosis infection. Health Technol Assess 2007;11:1-196.

41. Pai M, Flores LL, Hubbard A, et al. Nucleic acid amplification tests in the diagnosis of tuberculous pleuritis: a systematic review and meta-analysis. BMC Infect Dis 2004;4:6.

42. Yang WB, Liang QL, Ye ZJ, et al. Cell origins and diagnostic accuracy of interleukin 27 in pleural effusions. PloS One 2012;7:e40450.

43. Wu YB, Ye ZJ, Qin SM, et al. Combined detections of interleukin 27, interferon-gamma, and adenosine deaminase in pleural effusion for diagnosis of tuberculous pleurisy. Chin Med J 2013;126:3215-21.

44. Sun M, Yan D, Jiang S, et al. [Diagnostic value of interleukin-27 in tuberculous pleural effusion]. Zhonghua Yi Xue Za Zhi 2014;94:2641-4.

45. Valdés L, San José E, Ferreiro L, et al. Interleukin 27 could be useful in the diagnosis of tuberculous pleural effusions. Respir Care 2014;59:399-405.

46. Skouras VS, Magkouta SF, Psallidas I, et al. Interleukin-27 improves the ability of adenosine deaminase to rule 
out tuberculous pleural effusion regardless of pleural tuberculosis prevalence. Infect Dis (Lond) 2015;47:477-83.

47. Hooper C, Lee YC, Maskell N, et al. Investigation of a unilateral pleural effusion in adults: British Thoracic Society Pleural Disease Guideline 2010. Thorax 2010;65 Suppl 2:ii4-17.

48. Koegelenberg CF, Bolliger CT, Theron J, et al. Direct comparison of the diagnostic yield of ultrasoundassisted Abrams and Tru-Cut needle biopsies for pleural tuberculosis. Thorax 2010;65:857-62.

49. Patiala J. Initial tuberculous pleuritis in the Finnish armed forces in 1939-1945 with special reference to eventual postpleuritic tuberculosis. Acta Tuberc Scand Suppl 1954;36:1-57.

50. Roper WH, Waring JJ. Primary serofibrinous pleural effusion in military personnel. Am Rev Tuberc 1955;71:616-34.

51. Tani P, Poppius H, Maekipaja J. Cortisone therapy for exudative tuberculous pleurisy in the light of a follow-up study. Acta Tuberc Pneumol Scand 1964;44:303-9.

52. World Health Organization. Guidelines for treatment of tuberculosis, Fourth edition, 2010. Available online: http://apps.who.int/iris/bitstre am/10665/44165/1/9789241547833_eng.pdf?ua=1, Accessed 18 April 2016.

53. Horsburgh CR Jr, Barry CE 3rd, Lange C. Treatment of Tuberculosis. N Engl J Med 2015;373:2149-60.

54. Volmink J, Garner P. Directly observed therapy for treating tuberculosis. Cochrane Database Syst Rev 2007;(4):CD003343.

55. Gandhi NR, Nunn P, Dheda K, et al. Multidrug-resistant and extensively drug-resistant tuberculosis: a threat to global control of tuberculosis. Lancet 2010;375:1830-43.

56. Zhao Y, Xu S, Wang L, et al. National survey of drug-resistant tuberculosis in China. N Engl J Med 2012;366:2161-70.

57. Jindani A, Harrison TS, Nunn AJ, et al. High-dose rifapentine with moxifloxacin for pulmonary tuberculosis. N Engl J Med 2014;371:1599-608.

58. Lange C, Abubakar I, Alffenaar JW, et al. Management of patients with multidrug-resistant/extensively drug-resistant tuberculosis in Europe: a TBNET consensus statement. Eur Respir J 2014;44:23-63.

59. Ahuja SD, Ashkin D, Avendano M, et al. Multidrug resistant pulmonary tuberculosis treatment regimens and patient outcomes: an individual patient data meta-analysis of 9,153 patients. PLoS Med 2012;9:e1001300.

60. Lai YF, Chao TY, Wang YH, et al. Pigtail drainage in the treatment of tuberculous pleural effusions: a randomised study. Thorax 2003;58:149-51.

61. Chung CL, Chen CH, Yeh CY, et al. Early effective drainage in the treatment of loculated tuberculous pleurisy. Eur Respir J 2008;31:1261-7.

62. Cases Viedma E, Lorenzo Dus MJ, Gonzalez-Molina A, et al. A study of loculated tuberculous pleural effusions treated with intrapleural urokinase. Respir Med 2006;100:2037-42.

63. Galarza I, Canete C, Granados A, et al. Randomised trial of corticosteroids in the treatment of tuberculous pleurisy. Thorax 1995;50:1305-7.

64. Wyser C, Walzl G, Smedema JP, et al. Corticosteroids in the treatment of tuberculous pleurisy. A doubleblind, placebo-controlled, randomized study. Chest 1996;110:333-8.

65. Lee CH, Wang WJ, Lan RS, et al. Corticosteroids in the treatment of tuberculous pleurisy. A double-blind, placebocontrolled, randomized study. Chest 1988;94:1256-9.

66. Engel ME, Matchaba PT, Volmink J. Corticosteroids for tuberculous pleurisy. Cochrane Database Syst Rev 2007;(4):CD001876.

67. Elliott AM, Luzze H, Quigley MA, et al. A randomized, double-blind, placebo-controlled trial of the use of prednisolone as an adjunct to treatment in $\mathrm{HIV}$-1-associated pleural tuberculosis. J Infect Dis 2004;190:869-78.

68. Lawn SD, Meintjes G, McIlleron H, et al. Management of HIV-associated tuberculosis in resource-limited settings: a state-of-the-art review. BMC Med 2013;11:253.

69. Uthman OA, Okwundu C, Gbenga K, et al. Optimal Timing of Antiretroviral Therapy Initiation for HIVInfected Adults With Newly Diagnosed Pulmonary Tuberculosis: A Systematic Review and Meta-analysis. Ann Intern Med 2015;163:32-9.

70. Al-Majed SA. Study of paradoxical response to chemotherapy in tuberculous pleural effusion. Respir Med 1996;90:211-4.

71. Sahn SA, Iseman MD. Tuberculous empyema. Semin Respir Infect 1999;14:82-7.

72. Zarić B, Kuruc V, Milovancev A, et al. Differential diagnosis of tuberculous and malignant pleural effusions: what is the role of adenosine deaminase? Lung 2008;186:233-40.

Cite this article as: Zhai K, Lu Y, Shi HZ. Tuberculous pleural effusion. J Thorac Dis 2016;8(7):E486-E494. doi: 10.21037/ jtd.2016.05.87 